\title{
Investigating the association between radiological images and the pathology of rectal cancer treated with neoadjuvant chemotherapy
}

\author{
SATOKO MOROHASHI ${ }^{1}$, HAJIME MOROHASHI ${ }^{2}$, HIROKO SEINO ${ }^{3}$, \\ TADASHI YOSHIZAWA ${ }^{1}$, TOSHIHIRO HAGA ${ }^{1}$, SHINTARO GOTO ${ }^{1}$, YUNYAN WU ${ }^{1}$, \\ YOSHIYUKI SAKAMOTO $^{2}$, KENICHI HAKAMADA ${ }^{2}$ and HIROSHI KIJIMA ${ }^{1}$
}

Department of ${ }^{1}$ Pathology and Bioscience and ${ }^{2}$ Gastroenterological Surgery, Hirosaki University Graduate School of Medicine, Hirosaki, Aomori 036-8562; ${ }^{3}$ Department of Radiology, Aomori National Hospital, Namioka, Aomori 038-1331, Japan

Received February 20, 2019; Accepted June 18, 2019

DOI: $10.3892 / \mathrm{mco} .2019 .1931$

\begin{abstract}
In patients with rectal cancer treated with neoadjuvant chemotherapy (NAC), differences are often observed between high and low radiological image reduction effects. It may be suggested that high radiological image reduction indicates a beneficial response to chemotherapy. However, the pathological investigation of the differences between high and low radiological cancer volume reduction cases remains limited. In the current study, a total of 50 patients with rectal cancer, treated with NAC, were examined. The approximate pathological primary cancer area and the radiological cancer volume reduction ratio were measured using $\mathrm{CT}$ and/or MRI imaging and the donut-shaped measurement method. Immunostaining of cytokeratin AE1/AE3 was performed to quantitatively measure the cancer cell mass in the largest section of rectal cancer. Cytokeratin AE1/AE3-stained area $(\mathrm{P}=0.04)$, mitosis $(\mathrm{P}=0.0027)$ and radiological donut-shaped images after NAC $(\mathrm{P}=0.010)$ were lower in the high radiological cancer volume reduction ratio group compared with the low radiological cancer volume reduction ratio group. These findings indicate that the radiological images had some ability to determine the treatment effect and clinicopathological characteristics of patients with rectal cancer treated with NAC.
\end{abstract}

\section{Introduction}

In Japan, rectal cancer is the seventh most common cause of cancer-related death in men and the ninth highest in

Correspondence to: Dr Satoko Morohashi, Department of Pathology and Bioscience, Hirosaki University Graduate School of Medicine, 5 Zaifu-cho, Hirosaki, Aomori 036-8562, Japan

E-mail: msatoko@hirosaki-u.ac.jp; hkijima@hirosaki-u.ac.jp

\section{Abbreviations: NAC, neoadjuvant chemotherapy}

Key words: rectal cancer, neoadjuvant chemotherapy, radiological images, pathology, reduction ratio women (1). After neoadjuvant chemoradiotherapy treatment, rectal cancer can be controlled, distant recurrence can be prevented and disease-free survival and overall survival can be increased (2-5). Radiological imaging is often performed to assess the therapeutic effect of chemoradiotherapy during treatment. A recent study demonstrated the therapeutic effect and increased disease-free survival exhibited by chemoradiotherapy using computed tomography (CT) (6). In Japan, neoadjuvant chemotherapy (NAC) is accessible and is a promising treatment option for locally advanced rectal cancer (7). However, long-term bowel dysfunction has been reported to occur at a higher rate in irradiated patients compared with patients who undergo surgery alone (8). Radiotherapy is able to control local recurrence; however, radiation can induce fibrosis of the rectal wall, and may also cause proctitis, which can deteriorate anorectal function $(9,10)$. The direct radiation damage to the internal anal sphincter and nerve can cause anal sphincter dysfunction $(11,12)$. Therefore, identification of patients who would be susceptible to chemotherapy may enhance their quality of life and avoid these adverse effects. The current study aimed to compare the radiological images and pathology of rectal cancer treated with NAC, and elucidate its clinicopathological characteristics. The present study assessed the clinicopathological characteristics of rectal cancer cases in high and low radiological cancer volume reduction ratio groups after NAC.

\section{Patients and methods}

Patient samples. A retrospective assessment was conducted of the CT and/or MRI of 50 surgically resected rectal cancer lesions obtained prior to and following NAC from 2015 to 2018 at Hirosaki University Hospital (Hirosaki, Japan). Patient clinicopathological characteristics are presented in Table I. Informed consent was obtained from each patient regarding the use of their clinical records and pathological specimens. The patients underwent surgery from June 2015 to June 2018. The rectal cancer cases that exhibited metastases to other organs were excluded. Table II presents the chemotherapy regimens and the number of cycles performed on the patients prior to surgical resection. Clinical stage (cStage) was graded prior to and following NAC and pathological stage (pStage) 
was also graded based on the American Joint Committee on Cancer (AJCC) staging manual (13) (Table III).

Pathological examinations of primary cancer. Sections were obtained from every $5 \mathrm{~mm}$ at a cancer site, and all parts of each cancer lesion were sampled. For the histopathological examination, rectal cancer specimens were routinely $10 \%$ formalin-fixed (formalin fixation time, 48-120 h, room temperature), paraffin-embedded, thinly sectioned $(4 \mu \mathrm{m})$, and stained with hematoxylin and eosin (H\&E: Hematoxylin $20 \mathrm{~min}$ and eosin $3 \mathrm{~min}$ at room temperature, usually $\left.25^{\circ} \mathrm{C}\right)$. Pathological tumor stage (pT) and pathological node stage $(\mathrm{pN})$ were graded according to the AJCC staging manual (13). The pT, pN and pStage were each categorized into two groups (pT0-1 and pT2-4; pN0 and pN1-2; and pStage0-I and pStageII-III). Cancer lesions were graded based on histological analysis of the invasion of vessels (lymphatic invasion and venous invasion) and lymph node metastasis ( $\mathrm{pN} 0, \mathrm{pN} 1$ and $\mathrm{pN} 2$ ). Lymphatic invasion criteria were as follows: Ly0, no invasion; ly1, minimal invasion; ly2, moderate invasion; and ly3, severe invasion. Venous invasion criteria were: V0, no invasion; v1, minimal invasion; v2, moderate invasion; and v3, severe invasion. The lymphatic invasion and venous invasion were grouped into High (ly2, ly3/v2 and v3) and Low (ly0, ly1/v0 and v1) categories. The histological type was classified into tubular adenocarcinoma (well-differentiated type), tubular adenocarcinoma (moderately differentiated type), poorly differentiated type and mucinous adenocarcinoma. Mitosis was defined as the sum of three hotspots (x400) where mitosis was observed using a light microscope. The entire cancer tissues were scanned at low-magnification using a light microscope, and hot spots were selected; hot spots are representative areas including the highest number of mitotic cells in a field of light microscope $(\mathrm{x} 400)$. We counted the mitosis of cancer in each hot spot in a field of the light microscope (x400). The macro diameter was defined as the macroscopic cancer size $(\mathrm{mm})$.

Evaluation of cancer area on radiological images. Using DICOM data (EV Insite $\mathrm{R}$ version 3.4.0.0 from PSP Corporation or ShadeQuest/ViewR V1.24 from Yokogawa Medical Solutions) of CT and/or MRI images, the primary rectal cancer areas were calculated prior to and following NAC. CT scans were performed prior to and following NAC in most cases. In cases which CT could not be perform, MRI was performed instead. The radiological donut-shaped image was considered to be the cancer area, due to the precise identification of the tumor being challenging. Fig. 1 presents the whole intestinal wall at the dimension showing the greatest cancer area. The radiological donut-shaped image was defined as the area which remained following subtraction of the area of the gut lumen from the area enclosed by the perimeter of the intestinal tract at the dimension showing the greatest cancer area. The radiological cancer volume reduction ratio was also assessed using the radiological donut-shaped image prior to and following NAC as follows: Radiological cancer volume reduction ratio $(\%)=(1$-radiological donut-shaped image following NAC/radiological donut-shaped image before NAC) $\mathrm{x} 100$.
Residual cancer area on H\&E staining images. Whole tissue sections were examined in the current study. All slides comprising the maximal cancer section were examined. The pathological cancer area and pathological changed area were marked on the selected H\&E stained slides using a permanent marker (Fig. 2). Black permanent marker was used to indicate the pathological cancer area, and blue permanent marker was used to indicate the pathological changed area. The pathological cancer area was defined as the region in which cancer cells were distributed, including the region of fibrous stroma and inflammatory cells surrounding the cancer cells. Conversely, the pathological changed area was defined as the region in which cancer cells were not distributed. The pathological changed area was indicated by the disappearance of cancer cells following NAC, which also included granulated tissue, inflammatory cells and fibrosis. Sections were scanned using light microscopy at a low magnification (x12.5). Composite images were created so that one preparation could be seen in one image. The composition of images were created in Adobe Photoshop CC2014 (Adobe Systems, Inc.). Furthermore, the pathological cancer and pathological changed areas, which were marked using permanent marker, were measured using ImageJ software [Java 1.6.0_24 (64-bit); National Institutes of Health; http://imagej.nih.gov/ij; Fig. 2] (14). The pathological total area was calculated as the sum of the pathological cancer area and the pathological changed area.

Immunohistochemistry. Immunohistochemistry was performed on deparaffinized tissue sections using the standard avidin-biotin-peroxidase complex method with an automated immunostainer (Benchmark XT; Ventana Medical Systems, Inc.). The formalin fixation conditions were the same as those used for $\mathrm{H} \& \mathrm{E}$ staining. All slides containing the maximal cancer section were subjected immunostaining for the detection of Cytokeratin AE1/AE3. The antibody was used under the following conditions: Cytokeratin AE1/AE3 [M3515; dilution 1:100; or IS053 (Ready-to-Use); both Dako, Agilent Technologies, Inc.]. iVIEW DAB Universal kit (Roche Diagnostics KK), which includes a secondary antibody, inhibitor and DAB reagent, was used according to the manufacturer's protocol. The primary antibody was reacted for $16 \mathrm{~min}$ at room temperature (usually $25^{\circ} \mathrm{C}$ ). The cancer cells were then quantified by measuring the cytokeratin AE1/AE3-stained area of the rectal cancer. Specifically, the immunostained sections were scanned using light microscopy at a low magnification (x12.5). All pieces from the immunostained sections were combined into a single figure using Adobe Photoshop software. The color deconvolution plugin was used to obtain a binarization image using Image J software [Java 1.6.0_24 (64-bit)]. The cytokeratin AE1/AE3-stained area was outlined in black using ImageJ software and the cancer area was cropped out to remove the pathologically non-cancerous area. Subsequently, cytokeratin AE1/AE3-stained cancer cells were assessed using ImageJ software (Fig. 3).

Statistical analyses. Statistical comparisons were performed between high and low cancer volume reduction ratio groups, on radiological images, using a Pearson's Chi-square test. When the expected value was $<5$, a Fisher's test was used instead. The between-group statistical comparisons were analyzed 
Table I. Clinicopathological characteristics of 50 rectal cancer cases, treated with neoadjuvant chemotherapy.

\begin{tabular}{|c|c|}
\hline Clinicopathological feature & $\mathrm{V}$ \\
\hline Age, mean (range), years & $62.7(35-7$ \\
\hline \multicolumn{2}{|l|}{ Sex, n $(\%)$} \\
\hline Male & $38(76)$ \\
\hline Female & $12(24)$ \\
\hline \multicolumn{2}{|l|}{ Location, n (\%) } \\
\hline $\mathrm{Ra}$ & $2(4)$ \\
\hline $\mathrm{RaRb}$ & $1(2)$ \\
\hline $\mathrm{RaRbP}$ & $1(2)$ \\
\hline $\mathrm{Rb}$ & $43(86)$ \\
\hline $\mathrm{RbP}$ & $3(6)$ \\
\hline \multicolumn{2}{|l|}{ Pathological T stage, n (\%) } \\
\hline pT0 & $6(12)$ \\
\hline pT1 & $3(6)$ \\
\hline pT2 & $13(26)$ \\
\hline pT3 & $23(46)$ \\
\hline pT4 & $5(10)$ \\
\hline \multicolumn{2}{|l|}{ Pathological N stage, n (\%) } \\
\hline pNO & $35(70)$ \\
\hline $\mathrm{pN} 1$ & $13(26)$ \\
\hline $\mathrm{pN} 2$ & $2(4)$ \\
\hline \multicolumn{2}{|l|}{ Histology, n (\%) } \\
\hline $\begin{array}{l}\text { Tubular adenocarcinoma, well } \\
\text { differentiated type }\end{array}$ & $10(20)$ \\
\hline $\begin{array}{l}\text { Tubular adenocarcinoma, moderately } \\
\text { differentiated type }\end{array}$ & $33(66)$ \\
\hline Poorly differentiated type & $0(0)$ \\
\hline Mucinous adenocarcinoma & $1(2)$ \\
\hline N.A. & $6(12)$ \\
\hline \multicolumn{2}{|l|}{ Lymphatic invasion, $\mathrm{n}(\%)$} \\
\hline ly0 & $21(42)$ \\
\hline ly1 & $19(38)$ \\
\hline ly 2 & $8(16)$ \\
\hline ly3 & $2(4)$ \\
\hline \multicolumn{2}{|l|}{ Venous invasion, $\mathrm{n}(\%)$} \\
\hline $\mathrm{v0}$ & $11(22)$ \\
\hline $\mathrm{v} 1$ & $9(18)$ \\
\hline v2 & $22(44)$ \\
\hline v3 & $8(16)$ \\
\hline
\end{tabular}

Number of dissected lymph nodes, 27.48 (3-59) mean (range)

Pathological cancer area, mean

(range), $\mathrm{mm}^{2}$

Pathological changed area, mean

(range), $\mathrm{mm}^{2}$

Pathological total area, mean

(range), $\mathrm{mm}^{2}$

Cytokeratin AE1/AE3-stained area, mean (range), $\mathrm{mm}^{2}$

Mitosis, mean (range)

$168.1(0-794.3)$

$38.3(0.8-235.6)$

$206.3(10.461-811.125)$

$49.4(0-231.7)$

$20.5(0-69.0)$

$44.6(14.0-108.0)$
Table I. Continued.

\begin{tabular}{lc}
\hline Clinicopathological feature & Value \\
\hline $\begin{array}{l}\text { Interval from CT/MRI before NAC to } \\
\text { NAC start date, mean, days }\end{array}$ & 18.42 \\
$\begin{array}{l}\text { Interval from CT/MRI after NAC to } \\
\text { surgery date, mean, days }\end{array}$ & 26.28 \\
$\begin{array}{l}\text { Radiological donut-shaped image } \\
\text { before NAC, mean (range), mm }\end{array}$ & $1293(245.8-3596.4)$ \\
$\begin{array}{l}\text { Radiological donut-shaped image } \\
\text { after NAC, mean (range), mm }\end{array}$ & $958.8(103.0-1959.8)$ \\
Cancer volume reduction ratio on & \\
CT/MRI, mean (range), $\%$ & $23(-44.8-68.1)$
\end{tabular}

Ra, upper rectum, the portion of the large intestine located between the inferior border of the second sacral vertebra and the level of the peritoneal reflection; $\mathrm{Rb}$, lower rectum, the portion of the large intestine located between the peritoneal reflection and the superior border of the puborectal muscle; $\mathrm{P}$, anal canal, the tubular portion that extends from the superior border of the puborectal muscle to the anal verge; T, tumor; N, node; NAC, neoadjuvant chemotherapy; N.A., not available.

Table II. Chemotherapy regimens and the mean number of cycles.

\begin{tabular}{|c|c|c|}
\hline Chemotherapy regimen & $\mathrm{n}(\%)$ & Mean cycles \\
\hline FOLFOX + Pmab & $1(2)$ & 6.0 \\
\hline mFOLFOX6 & $1(2)$ & 6.0 \\
\hline mFOLFOX6+Bv & $2(4)$ & 6.0 \\
\hline SOX & $38(76)$ & 2.9 \\
\hline $\mathrm{SOX}+\mathrm{Bv}$ & $7(14)$ & 4.0 \\
\hline $\mathrm{SOX}+\mathrm{Bv} \rightarrow \mathrm{FOLFIRI}+\mathrm{Bv}$ & $1(2)$ & 5.0 \\
\hline \multicolumn{3}{|c|}{$\begin{array}{l}\text { FOLFOX, infusional 5FU }+ \text { l-leucovorin }+ \text { oxaliplatin; } \\
\text { mFOLFOX, modified FOLFOX, comprising modified infusional } \\
5 \text { FU + 1-leucovorin }+ \text { oxaliplatin; Pmab, panitumumab; Bv, } \\
\text { bevacizumab; SOX, S- }+ \text { oxaliplatin; FOLFIRI, infusional } \\
\text { 5FU + 1-leucovorin }+ \text { irinotecan; SOX }+ \text { Bv } \rightarrow \text { FOLFIRI+Bv, } \\
\text { SOX + Bv } 3 \text { cycles followed by FOLFIRI }+ \text { Bv } 2 \text { cycles. }\end{array}$} \\
\hline
\end{tabular}

using the Wilcoxon rank-sum test for non-normally distributed continuous data and a two sample t-test for normally distributed data. $\mathrm{P}<0.05$ was considered to indicate a statistically significant result. All statistical analyses were performed using R (http://www.r-project.org).

\section{Results}

Clinicopathological features. Table I summarizes the clinicopathological characteristics of the 50 rectal cancer cases treated with NAC. The average age of the patients was 62.7 years. There were 38 male patients $(76 \%)$ and 12 female patients $(24 \%)$. The locations of the rectal cancer were $\mathrm{Ra}$ ( 2 patients, $4 \%$ ), RaRb (1 patient, $2 \%$ ), RaRbP 
A

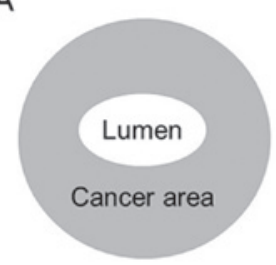

B

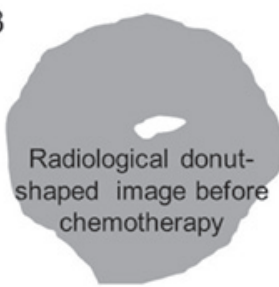

C

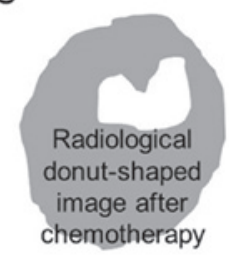

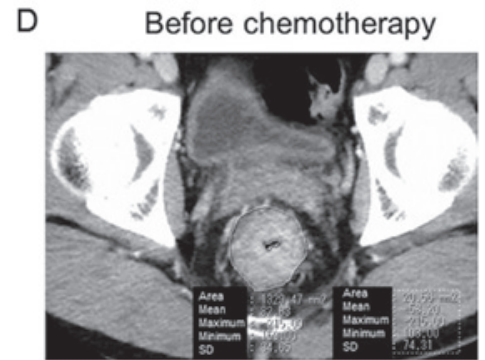

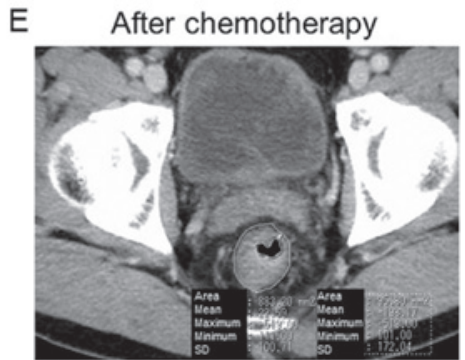

Figure 1. Assessment of a cancer volume on a radiological image. Schematics of (A) the components of the radiological donut-shaped image (gray area), (B) before and (C) after NAC. CT imaging of the radiological donut-shaped image (D) before and (E) after NAC. NAC, neoadjuvant chemotherapy; $\mathrm{SD}$, standard deviation.

Table III. cStage before and after NAC, and the pStage.

\begin{tabular}{lccr}
\hline Stage & $\begin{array}{c}\text { cStage before } \\
\text { NAC, n (\%) }\end{array}$ & $\begin{array}{c}\text { cStage after } \\
\text { NAC, n (\%) }\end{array}$ & $\begin{array}{c}\text { pStage, } \\
\mathrm{n}(\%)\end{array}$ \\
\hline 0 & $0(0)$ & $1(2)$ & $6(12)$ \\
I & $0(0)$ & $7(14)$ & $14(28)$ \\
II & $19(38)$ & $24(48)$ & $15(30)$ \\
III & $31(62)$ & $18(36)$ & $15(30)$ \\
\hline
\end{tabular}

cStage, clinical stage; NAC, neoadjuvant chemotherapy; pStage, pathological stage.

(1 patient, $2 \%$ ), Rb (43 patients, $86 \%$ ) and $\mathrm{RbP}$ (3 patients, 6\%). Pathological T stages were pT0 (6 patients, 12\%), pT1 (3 patients, $6 \%$ ), pT2 (13 patients, 26\%), pT3 (23 patients, $46 \%)$ and pT4 (5 patients, 10\%). Pathological N stages were pN0 (35 patients, 70\%), pN1 (13 patients, 26\%) and pN2 (2 patients, $4 \%$ ). Histology was tubular adenocarcinoma, well differentiated type (10 patients, 20\%), moderately differentiated type (33 patients, 66\%), poorly differentiated (0 patient, $0 \%$ ), mucinous adenocarcinoma (1 patient, $2 \%$ ) and not available (N.A.; 6 patients, $12 \%$ ). Lymphatic invasion was as follows: Ly0, 21 patients $(42 \%)$; ly1, 19 patients (38\%); ly 2, 8 patients (16\%); and ly3, 2 patients (4\%). Venous invasion was as follows: V0, 11 patients $(22 \%)$; v1, 9 patients (18\%); v2, 22 patients (44\%); and v3, 8 patients $(16 \%)$. A total of 6 patients achieved a complete pathological response. All 6 complete pathological response cases were graded after NAC as follows: pT0, pN0, N.A. (histology), ly0 and $\mathrm{v} 0$. The 6 complete response patients did not have any cancer cells, so all 6 cases were classified as histologic type N.A. The mean number of dissected lymph nodes was 27.48. The mean pathological cancer area was $168.1 \mathrm{~mm}^{2}$. The mean pathological changed area and pathological total area were 38.3 and $206.3 \mathrm{~mm}^{2}$, respectively. The
cytokeratinAE1/AE3-stained area was $49.4 \mathrm{~mm}^{2}$. The mean intervals from CT/MRI before NAC to the NAC start date, and from CT/MRI after NAC to the surgery date was 18.42 and 26.28 days, respectively. The mean radiological donut-shaped image before and after NAC were 1,293 and $958.8 \mathrm{~mm}^{2}$, respectively. The mean cancer volume reduction ratio on CT/MRI was $23 \%$.

cStage prior to, and cStage and pStage following, NAC. Table III presents the cStage prior to, and cStage and pStage following NAC. No cStage0 or cStageI cases were classified prior to NAC; however, some Stage0 and StageI cases were classified following NAC. There were many cStageIII cases prior to NAC; however, the number of patients with StageIII decreased after NAC (cStage and pStage).

Definition and characteristics of the high and low radiological cancer volume reduction groups. In the current study, the average value of the radiological cancer volume reduction ratio was $23 \%$. Therefore, $\geq 23 \%$ was considered as the high radiological cancer volume reduction group, whereas $<23 \%$ was regarded as the low radiological cancer volume reduction ratio group. These patient groups were used for subsequent analysis.

A total of 25 patients were included in the high radiological cancer volume reduction group, and 25 patients were included in the low radiological cancer volume reduction group. The cytokeratin AE1/AE3-stained area $(\mathrm{P}=0.04)$, mitosis $(\mathrm{P}=0.0027)$ and radiological donut-shaped image after NAC $(\mathrm{P}=0.010)$ were significantly lower in the high radiological cancer volume reduction ratio group compared with the low radiological cancer volume reduction group (Table IV). There was no difference between the two groups regarding age, sex, pT, $\mathrm{pN}$, pStage, lymphatic invasion, venous invasion, pathological cancer area, pathological changed area, pathological total area, macro diameter and donut-shaped image before chemotherapy. All 6 complete pathological response cases were in the high radiological cancer volume reduction ratio group. 


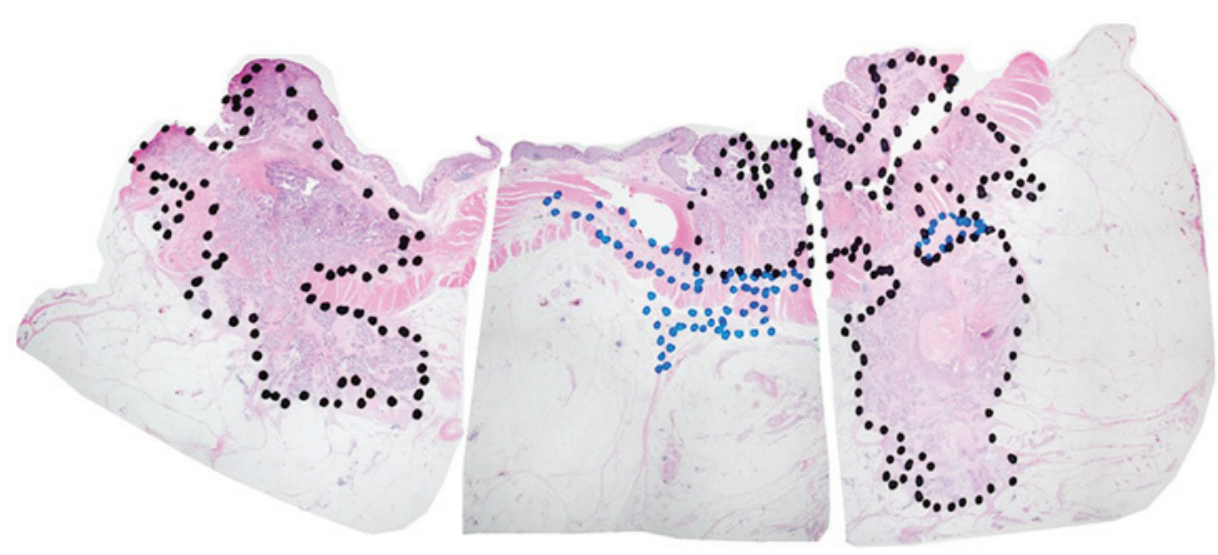

Figure 2. Measurement of the pathological cancer area/pathological changed area on hematoxylin and eosin staining images (magnification, x12.5). Black permanent marker, the pathological cancer area; blue permanent marker, the pathological changed area.
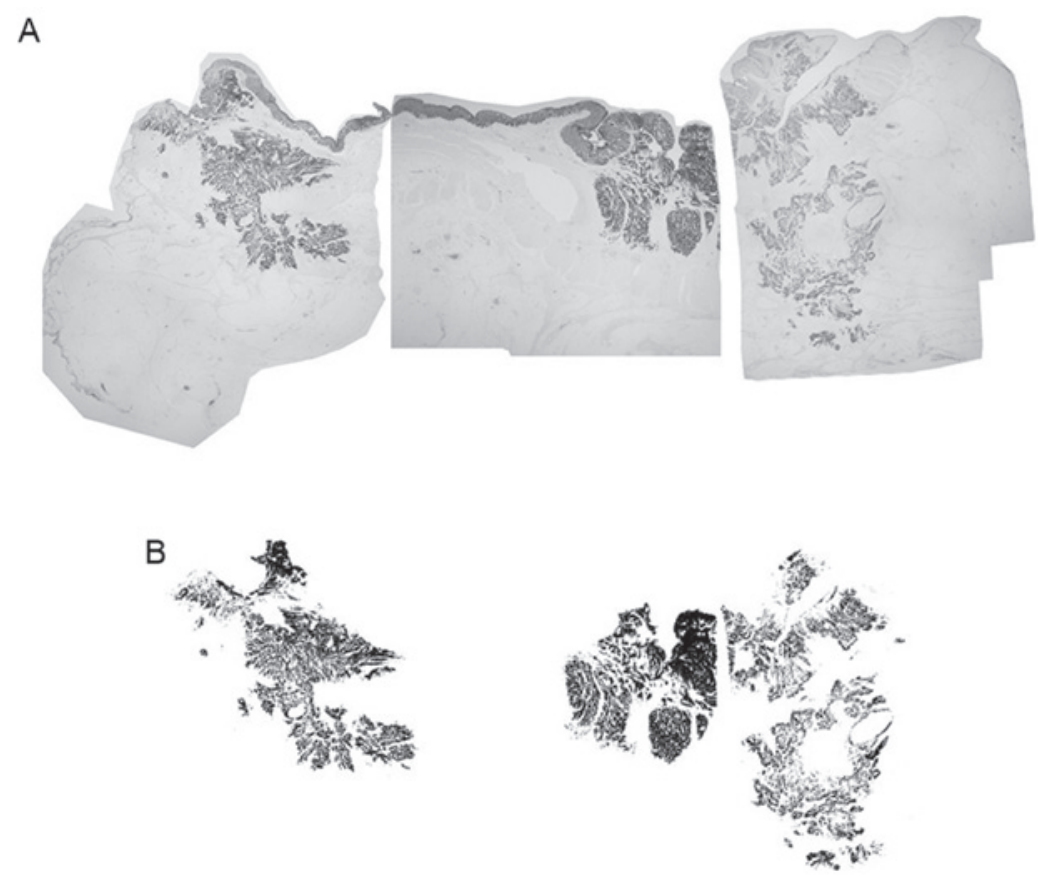

Figure 3. Cytokeratin AE1/AE3-stained area. (A) Immunohistochemistry and (B) binary image for cytokeratin AE1/AE3 staining (magnification, x12.5).

\section{Discussion}

A number of studies have demonstrated the correlation between pathological therapeutic effect and prognosis in rectal cancer after preoperative chemoradiotherapy $(3,15)$. However, to the best of our knowledge, no study has compared the radiological images and pathology of rectal cancer cases treated with NAC. In the current study, clinicopathological differences were compared between high and low radiological cancer volume reduction ratio groups in patients with rectal cancer treated with NAC. The rectal cancer area is difficult to measure accurately as a luminal organ using CT and/or MRI, so the radiological donut-shaped image of the rectum was used, at the maximum cancer size prior to and following NAC. The radiological cancer reduction ratio was then calculated from this analysis.

The cytokeratin AE1/AE3-stained area was smaller in the high radiological cancer volume reduction ratio group compared with the low radiological cancer volume reduction ratio group. This result may suggest that the radiological cancer volume reduction ratio can be used to estimate the approximate cancer cell mass following NAC treatment. There was no previous indication that CT and/or MRI imaging could predict cancer cell mass following NAC. CT and MRI are two commonly used clinical non-invasive imaging techniques. In the current study, CT and MRI were used to determine if the tumor size decreased following NAC treatment, and the results indicated that tumor cell mass reduced following NAC. Additionally, mitosis was lower in the high radiological cancer volume reduction ratio group compared with the low radiological cancer volume reduction ratio group. High radiological cancer volume reduction ratio may indicate low cancer cell proliferation. The association exhibited between radiological images and pathology after NAC may potentially influence the management of patients with rectal cancer. Radiological cancer volume reduction ratio could potentially be used to indicate if NAC treatment is pathologically effective 
Table IV. Clinicopathological characteristics of the low and high radiological cancer volume reduction ratio groups.

\begin{tabular}{|c|c|c|c|}
\hline Clinicopathological feature & $\begin{array}{l}\text { Low radiological } \\
\text { cancer volume reduction } \\
\text { ratio group }(n=25)\end{array}$ & $\begin{array}{l}\text { High radiological } \\
\text { cancer volume reduction } \\
\text { ratio group }(n=25)\end{array}$ & P-value \\
\hline Age, median, years & 65 & 66 & 0.85 \\
\hline Sex, male/female & $20(80) / 5(20)$ & $18(72) / 7(28)$ & 0.51 \\
\hline Pathological T stage, pT0-1/pT2-4 & $2(8) / 23(92)$ & $7(28) / 18(72)$ & 0.069 \\
\hline Pathological N stage, pN0/pN1-2 & $16(64) / 9(36)$ & $19(76) / 6(24)$ & 0.355 \\
\hline Pathological stage, pStage0-I/pStageII-III & $7(28) / 18(72)$ & $13(52) / 12(48)$ & 0.083 \\
\hline Lymphatic invasion, high (ly2-3)/low (ly0-) & $8(32) / 17(68)$ & $2(8) / 23(92)$ & 0.074 \\
\hline Venous invasion, high (v2-3)/low (v0-1) & $18(72) / 7(28)$ & $12(48) / 13(52)$ & 0.083 \\
\hline Pathological cancer area, median, $\mathrm{mm}^{2}$ & 105 & 55.6 & 0.14 \\
\hline Pathological changed area, median, $\mathrm{mm}^{2}$ & 24.3 & 26.7 & 0.8 \\
\hline Pathological total area, median, $\mathrm{mm}^{2}$ & 137 & 76.2 & 0.22 \\
\hline CytokeratinAE1/AE3-stained area, median, $\mathrm{mm}^{2}$ & 42.8 & 14.3 & 0.04 \\
\hline Mitosis, median & 26 & 12 & 0.0027 \\
\hline Macro diameter, median, $\mathrm{mm}$ & 36 & 37 & 0.97 \\
\hline Radiological donut-shaped image before NAC, median & 1075.7 & 1259.8 & 0.095 \\
\hline Radiological donut-shaped image after NAC, median & 987.4 & 728.1 & 0.010 \\
\hline
\end{tabular}

T, tumor; N, node; NAC, neoadjuvant chemotherapy. Results are presented as n (\%), unless otherwise specified.

in patients with rectal cancer, and this may influence treatment decisions. The pathological cancer area and pathological changed areas were not observed to be directly associated with the radiological reduction ratio. This discrepancy could be attributed to radiologically reduced cancer volumes without pathological fibrosis/scar formation. In some patient cases, cancer tissues completely disappeared after NAC and marked histological changes (including fibrosis or scarring) were not identified, whereas tumor volumes declined in the radiological image. Due to this, the assessment of the pathological therapeutic effect is indicated to be subjective, which could also account for the pathological/radiological discrepancy. The radiological donut-shaped image after NAC was smaller in the high radiological cancer volume reduction ratio group. The radiological cancer volume reduction ratio was associated with the radiological donut-shaped image following NAC, irrespective of the image captured prior to NAC, suggesting that the preoperative size (prior to NAC) of the cancer area was not crucial, but the cancer area after NAC (radiological donut-shaped image following NAC) was crucial.

Dworak et al (16) proposed the grading of regression as a pathological feature of rectal cancer after preoperative chemoradiotherapy. Dworak's regression was graded from grade 0 to 4 . The grading of regression is focused on fibrosis in cancer and is a semiquantitative system that associates treatment effect with the amount of fibrosis. Reportedly, tumor regression grade (TRG) has prognostic significance after preoperative chemoradiotherapy in rectal cancer $(3,15)$. Mandard et al (17) reported TRG in esophageal cancer after preoperative therapy. TRG comprises five grades from TRG1 to TRG5 and is also a semiquantitative system that associates the amount of residual cancer with the amount of fibrosis. The present study examined pathological changed area as the treatment effect area. However, no significant differences were exhibited between the high and low radiological cancer volume reduction ratio groups in pathologically changed area. For preoperative chemoradiation and NAC in rectal cancer, histological therapeutic effects may differ. New histological therapeutic effect classifications are required for NAC in rectal cancer. CytokeratinAE1/AE3-stained area may be used to develop new histological therapeutic classifications.

In conclusion, the current study indicated an association between radiological images and the pathology of rectal cancer treated with NAC. Only 50 patient cases were examined; however, the results of the current study may facilitate the estimation of pathological factors in surgical specimens from radiological image examination, with the accumulation of further analysis in the future.

\section{Acknowledgements}

Not applicable.

\section{Funding}

The present study was supported by Grants-in-Aid for Science from The Ministry of Education, Culture, Sports, Science and Technology of Japan; The Hirosaki University Institutional Research and The Fund for the Promotion of International Scientific Research (grant no. 17H04057).

\section{Availability of data and materials}

The datasets used and/or analyzed during the current study are available from the corresponding author on reasonable request. 


\section{Authors' contributions}

SM wrote the manuscript and made substantial contribution to acquisition of data and analysis. HS, TY, TH, SG, YW, and HK provided substantial contributions to analysis and interpretation of data in the present study. HM, YS, and $\mathrm{KH}$ made substantial contributions to the acquisition of data. All authors read and approved the final manuscript.

\section{Ethics approval and consent to participate}

This study performed in accordance with the Declaration of Helsinki and was approved by the Ethics Committee of Hirosaki University Graduate School of Medicine (organization no. 2015-118).

\section{Patient consent for publication}

The patients provided written informed consent for the publication of any associated data and accompanying images.

\section{Competing interests}

The authors declare that they have no competing interests.

\section{References}

1. Hori M, Matsuda T, Shibata A, Katanoda K, Sobue T and Nishimoto H; Japan Cancer Surveillance Research Group: Cancer incidence and incidence rates in Japan in 2009: A study of 32 population-based cancer registries for the monitoring of cancer incidence in Japan (MCIJ) project. Jpn J Clin Oncol 45: 884-891, 2015.

2. Díaz-González JA, Calvo FA, Cortés J, García-Sabrido JL, Gómez-Espí M, Del Valle E, Muñoz-Jiménez F and Alvarez E: Prognostic factors for disease-free survival in patients with T3-4 or $\mathrm{N}^{+}$rectal cancer treated with preoperative chemoradiation therapy, surgery, and intraoperative irradiation. Int J Radiat Oncol Biol Phys 64: 1122-1128, 2006.

3. Rödel C, Martus P, Papadoupolos T, Füzesi L, Klimpfinger M, Fietkau R, Liersch T, Hohenberger W, Raab R, Sauer R and Wittekind C: Prognostic significance of tumor regression after preoperative chemoradiotherapy for rectal cancer. J Clin Oncol 23: 8688-8696, 2005.

4. Valentini V, Coco C, Picciocchi A, Morganti AG, Trodella L, Ciabattoni A, Cellini F, Barbaro B, Cogliandolo S, Nuzzo G, et al: Does downstaging predict improved outcome after preoperative chemoradiation for extraperitoneal locally advanced rectal cancer? A long-term analysis of 165 patients. Int J Radiat Oncol Biol Phys 53: 664-674, 2002.

5. Vecchio FM, Valentini V, Minsky BD, Padula GD, Venkatraman ES, Balducci M, Miccichè F, Ricci R, Morganti AG, Gambacorta MA, et al: The relationship of pathologic tumor regression grade (TRG) and outcomes after preoperative therapy in rectal cancer. Int J Radiat Oncol Biol Phys 62: 752-760, 2005.
6. Chee CG, Kim YH, Lee KH, Lee YJ, Park JH, Lee HS, Ahn S and Kim B: CT texture analysis in patients with locally advanced rectal cancer treated with neoadjuvant chemoradiotherapy: A potential imaging biomarker for treatment response and prognosis. PLoS One 12: e0182883, 2017.

7. Hasegawa S, Goto S, Matsumoto T, Hida K, Kawada K, Matsusue R, Yamaguchi T, Nishitai R, Manaka D, Kato S, et al: A multicenter phase 2 study on the feasibility and efficacy of neoadjuvant chemotherapy without radiotherapy for locally advanced rectal cancer. Ann Surg Oncol 24: 3587-3595, 2017

8. Peeters KC, van de Velde CJ, Leer JW, Martijn H, Junggeburt JM, Kranenbarg EK, Steup WH, Wiggers T, Rutten HJ and Marijnen CA: Late side effects of short-course preoperative radiotherapy combined with total mesorectal excision for rectal cancer: Increased bowel dysfunction in irradiated patients-a Dutch colorectal cancer group study. J Clin Oncol 23: 6199-6206, 2005.

9. Chen FC, Mackay JR, Woods RJ, Collopy BT, Fink RJ and Guiney MJ: Early experience with postoperative adjuvant chemoradiation for rectal carcinoma: Focus on morbidity. Aust N Z J Surg 65: 732-736, 1995.

10. Kollmorgen CF, Meagher AP, Wolff BG, Pemberton JH, Martenson JA and Illstrup DM: The long-term effect of adjuvant postoperative chemoradiotherapy for rectal carcinoma on bowel function. Ann Surg 220: 676-682, 1994.

11. Da Silva GM, Berho M, Wexner SD, Efron J, Weiss EG, Nogueras JJ, Vernava AM III, Connor JT and Gervaz P: Histologic analysis of the irradiated anal sphincter. Dis Colon Rectum 46: 1492-1497, 2003.

12. Nishizawa Y, Fujii S, Saito N, Ito M, Ochiai A, Sugito M, Kobayashi A and Nishizawa Y: The association between anal function and neural degeneration after preoperative chemoradiotherapy followed by intersphincteric resection. Dis Colon Rectum 54: 1423-1429, 2011.

13. Milburn Jessup J, Goldber RM, Asare EA, Benson ALB III, Brierley JD, Chang GJ, Chen V, Compton CC, De Nard P, Goodman KA, et al (eds): AJCC: AJCC cancer staging manual. In: Colon and Rectum 8th edition. Springer, Switzerland, pp251-274, 2017.

14. Abramoff MD, Magelhaes PJ and Ram SJ: Image Processing with ImageJ. Biophot Int 11: 36-42, 2004

15. Fokas E, Strobel P, Fietkau R, Ghadimi M, Liersch T, Grabenbauer GG, Hartmann A, Kaufmann M, Sauer R, Graeven U, et al: Tumor regression grading after preoperative chemoradiotherapy as a prognostic factor and individual-level surrogate for disease-free survival in rectal cancer. J Natl Cancer Inst 109, 2017.

16. Dworak O, Keilholz L and Hoffmann A: Pathological features of rectal cancer after preoperative radiochemotherapy. Int J Colorectal Dis 12: 19-23, 1997.

17. Mandard AM,Dalibard F, Mandard JC, Marnay J,Henry-Amar M, Petiot JF, Roussel A, Jacob JH, Segol P, Samama G, et al: Pathologic assessment of tumor regression after preoperative chemoradiotherapy of esophageal carcinoma. Clinicopathologic correlations. Cancer 73: 2680-2686, 1994.

This work is licensed under a Creative Commons Attribution-NonCommercial-NoDerivatives 4.0 International (CC BY-NC-ND 4.0) License. 